\title{
Congenital progressive polymorphic cataract caused by a mutation in the major intrinsic protein of the lens, MIP (AQP0)
}

\author{
Peter Francis, Vanita Berry, Shomi Bhattacharya, Anthony Moore
}

Institute of

Ophthalmology,

London, UK

P Francis

V Berry

S Bhattacharya

\section{Moorfields Eye}

Hospital, London, UK

P Francis

A Moore

Addenbrooke's Hospital, Cambridge, UK

A Moore

Correspondence to: Mr A T Moore, Moorfields Eye Hospital, City Road, London EC1V 2PD, UK atm22@hermes.cam.ac.uk

Accepted for publication 22 June 2000

Table 1 (a) Mapped loci for human autosomal dominant congenital cataract, (b) identified human congenital cataract mutations

\begin{tabular}{|c|c|c|c|c|}
\hline \multicolumn{2}{|l|}{$\begin{array}{l}\text { (a) } \\
\text { Phenotype }\end{array}$} & Locus & Inheritance & Reference \\
\hline \multicolumn{2}{|c|}{ Volkmann (pulverulent) } & $1 \mathrm{p} 36$ & $\mathrm{AD}$ & 20 \\
\hline \multicolumn{2}{|c|}{ Posterior polar } & $1 \mathrm{p} 36$ & $\mathrm{AD}$ & 12 \\
\hline \multicolumn{2}{|l|}{ Nuclear } & $12 \mathrm{q} 12-14.1$ & $\mathrm{AD}$ & 21 \\
\hline \multicolumn{2}{|l|}{ Anterior polar } & $14 \mathrm{q} 24$ & Translocation & 22 \\
\hline \multicolumn{2}{|l|}{ Unknown } & $16 \mathrm{p} 13.3$ & Translocation & 23 \\
\hline \multicolumn{2}{|l|}{ Marner } & $16 \mathrm{q} 22.1$ & $\mathrm{AD}$ & 24 \\
\hline \multicolumn{2}{|l|}{ Posterior polar } & $16 \mathrm{q} 22.1$ & $\mathrm{AD}$ & 24 \\
\hline \multicolumn{2}{|c|}{ Anterior polar } & $17 \mathrm{p} 13$ & $\mathrm{AD}$ & 25 \\
\hline \multicolumn{2}{|c|}{ Zonular sutural (lamellar) } & $17 \mathrm{q} 11-12$ & $\mathrm{AD}$ & 26 \\
\hline \multicolumn{2}{|c|}{ Cerulean } & $17 \mathrm{q} 24$ & $\mathrm{AD}$ & 27 \\
\hline \multicolumn{2}{|c|}{ Unknown } & I-blood group locus & AR & 28 \\
\hline \multicolumn{2}{|c|}{ Sutural (lamellar) } & Xpter-Xqter & $\mathrm{XR}$ & 293031 \\
\hline \multicolumn{5}{|l|}{ (b) } \\
\hline Locus & Gene & Protein & Mutation & Reference \\
\hline $1 \mathrm{q} 21-\mathrm{q} 25$ & GFA8 & Connexin 50 & missense & 7 \\
\hline $2 q 33-q 35$ & $C R Y G D$ & $\gamma \mathrm{D}$ crystallin & missense & 32 \\
\hline $2 \mathrm{q} 33-\mathrm{q} 35$ & $C R Y G C$ & $\gamma \mathrm{C}$ crystallin & missense & 33 \\
\hline $10 q 24-25$ & PITX3 & Pitx3 & missense & 34 \\
\hline $12 \mathrm{q} 14$ & $M I P$ & MIP/AQP0 & missense & 10 \\
\hline 13q11-q13 & $C X 46$ & Connexin 46 & missense & 9 \\
\hline $17 \mathrm{q} 11.1-\mathrm{q} 12$ & $C R Y B A 1$ & BA1, crystallin & splice site & 26 \\
\hline $21 \mathrm{q} 22.3$ & $C R Y A A$ & $\alpha$ crystallin & missense & 5 \\
\hline $22 \mathrm{q} 11.2$ & $C R Y B B 2$ & $\beta$ crystallin & chain termination & 35 \\
\hline $22 \mathrm{q} 11.2$ & $C R Y B B 2$ & $\beta$ crystallin & missense & 36 \\
\hline
\end{tabular}

Furthermore, this is the first clear evidence of allelic heterogeneity in this condition following the identification of a family with lamellar cataracts who have a different mutation within the MIP gene.

(Br f Ophthalmol 2000;84:1376-1379)

Abstract
Background-Congenital cataract, when
inherited as an isolated abnormality, is
phenotypically and genetically heteroge-
neous. Although there is no agreed no-
menclature for the patterns of cataract
observed, a recent study identified eight
readily identifiable phenotypes.
Methods-The Moorfields Eye Hospital
genetic eye clinic database was used to
identify a four generation family with iso-
lated autosomal dominant congenital
cataracts. All individuals (affected and
unaffected) underwent a full ophthalmic
assessment.
Results-The results of the molecular
linkage study identifying a missense mu-
tation in the gene encoding the major
intrinsic protein of the lens (MIP) have
been published elsewhere. Affected indi-
viduals had bilateral discrete progressive
punctate lens opacities limited to mid and
peripheral lamellae with additional asym-
metric polar opacification. One young
female had predominantly cortical cata-
ract and another had serpiginous nuclear
opacities.
Conclusions-This phenotype has not
been recorded in human families before
and has been termed polymorphic. The
pattern of opacification appears to reflect
the distribution of MIP in the lens.

Abstract
Background-Congenital cataract, when
inherited as an isolated abnormality, is
phenotypically and genetically heteroge-
neous. Although there is no agreed no-
menclature for the patterns of cataract
observed, a recent study identified eight
readily identifiable phenotypes.
Methods-The Moorfields Eye Hospital
genetic eye clinic database was used to
identify a four generation family with iso-
lated autosomal dominant congenital
cataracts. All individuals (affected and
unaffected) underwent a full ophthalmic
assessment.
Results-The results of the molecular
linkage study identifying a missense mu-
tation in the gene encoding the major
intrinsic protein of the lens (MIP) have
been published elsewhere. Affected indi-
viduals had bilateral discrete progressive
punctate lens opacities limited to mid and
peripheral lamellae with additional asym-
metric polar opacification. One young
female had predominantly cortical cata-
ract and another had serpiginous nuclear
opacities.
Conclusions-This phenotype has not
been recorded in human families before
and has been termed polymorphic. The
pattern of opacification appears to reflect
the distribution of MIP in the lens.

Abstract
Background-Congenital cataract, when
inherited as an isolated abnormality, is
phenotypically and genetically heteroge-
neous. Although there is no agreed no-
menclature for the patterns of cataract
observed, a recent study identified eight
readily identifiable phenotypes.
Methods-The Moorfields Eye Hospital
genetic eye clinic database was used to
identify a four generation family with iso-
lated autosomal dominant congenital
cataracts. All individuals (affected and
unaffected) underwent a full ophthalmic
assessment.
Results-The results of the molecular
linkage study identifying a missense mu-
tation in the gene encoding the major
intrinsic protein of the lens (MIP) have
been published elsewhere. Affected indi-
viduals had bilateral discrete progressive
punctate lens opacities limited to mid and
peripheral lamellae with additional asym-
metric polar opacification. One young
female had predominantly cortical cata-
ract and another had serpiginous nuclear
opacities.
Conclusions-This phenotype has not
been recorded in human families before
and has been termed polymorphic. The
pattern of opacification appears to reflect
the distribution of MIP in the lens.

Abstract
Background-Congenital cataract, when
inherited as an isolated abnormality, is
phenotypically and genetically heteroge-
neous. Although there is no agreed no-
menclature for the patterns of cataract
observed, a recent study identified eight
readily identifiable phenotypes.
Methods-The Moorfields Eye Hospital
genetic eye clinic database was used to
identify a four generation family with iso-
lated autosomal dominant congenital
cataracts. All individuals (affected and
unaffected) underwent a full ophthalmic
assessment.
Results-The results of the molecular
linkage study identifying a missense mu-
tation in the gene encoding the major
intrinsic protein of the lens (MIP) have
been published elsewhere. Affected indi-
viduals had bilateral discrete progressive
punctate lens opacities limited to mid and
peripheral lamellae with additional asym-
metric polar opacification. One young
female had predominantly cortical cata-
ract and another had serpiginous nuclear
opacities.
Conclusions-This phenotype has not
been recorded in human families before
and has been termed polymorphic. The
pattern of opacification appears to reflect
the distribution of MIP in the lens.

Abstract
Background-Congenital cataract, when
inherited as an isolated abnormality, is
phenotypically and genetically heteroge-
neous. Although there is no agreed no-
menclature for the patterns of cataract
observed, a recent study identified eight
readily identifiable phenotypes.
Methods-The Moorfields Eye Hospital
genetic eye clinic database was used to
identify a four generation family with iso-
lated autosomal dominant congenital
cataracts. All individuals (affected and
unaffected) underwent a full ophthalmic
assessment.
Results-The results of the molecular
linkage study identifying a missense mu-
tation in the gene encoding the major
intrinsic protein of the lens (MIP) have
been published elsewhere. Affected indi-
viduals had bilateral discrete progressive
punctate lens opacities limited to mid and
peripheral lamellae with additional asym-
metric polar opacification. One young
female had predominantly cortical cata-
ract and another had serpiginous nuclear
opacities.
Conclusions-This phenotype has not
been recorded in human families before
and has been termed polymorphic. The
pattern of opacification appears to reflect
the distribution of MIP in the lens.

Abstract
Background-Congenital cataract, when
inherited as an isolated abnormality, is
phenotypically and genetically heteroge-
neous. Although there is no agreed no-
menclature for the patterns of cataract
observed, a recent study identified eight
readily identifiable phenotypes.
Methods-The Moorfields Eye Hospital
genetic eye clinic database was used to
identify a four generation family with iso-
lated autosomal dominant congenital
cataracts. All individuals (affected and
unaffected) underwent a full ophthalmic
assessment.
Results-The results of the molecular
linkage study identifying a missense mu-
tation in the gene encoding the major
intrinsic protein of the lens (MIP) have
been published elsewhere. Affected indi-
viduals had bilateral discrete progressive
punctate lens opacities limited to mid and
peripheral lamellae with additional asym-
metric polar opacification. One young
female had predominantly cortical cata-
ract and another had serpiginous nuclear
opacities.
Conclusions-This phenotype has not
been recorded in human families before
and has been termed polymorphic. The
pattern of opacification appears to reflect
the distribution of MIP in the lens.

Abstract
Background-Congenital cataract, when
inherited as an isolated abnormality, is
phenotypically and genetically heteroge-
neous. Although there is no agreed no-
menclature for the patterns of cataract
observed, a recent study identified eight
readily identifiable phenotypes.
Methods-The Moorfields Eye Hospital
genetic eye clinic database was used to
identify a four generation family with iso-
lated autosomal dominant congenital
cataracts. All individuals (affected and
unaffected) underwent a full ophthalmic
assessment.
Results-The results of the molecular
linkage study identifying a missense mu-
tation in the gene encoding the major
intrinsic protein of the lens (MIP) have
been published elsewhere. Affected indi-
viduals had bilateral discrete progressive
punctate lens opacities limited to mid and
peripheral lamellae with additional asym-
metric polar opacification. One young
female had predominantly cortical cata-
ract and another had serpiginous nuclear
opacities.
Conclusions-This phenotype has not
been recorded in human families before
and has been termed polymorphic. The
pattern of opacification appears to reflect
the distribution of MIP in the lens.

Abstract
Background-Congenital cataract, when
inherited as an isolated abnormality, is
phenotypically and genetically heteroge-
neous. Although there is no agreed no-
menclature for the patterns of cataract
observed, a recent study identified eight
readily identifiable phenotypes.
Methods-The Moorfields Eye Hospital
genetic eye clinic database was used to
identify a four generation family with iso-
lated autosomal dominant congenital
cataracts. All individuals (affected and
unaffected) underwent a full ophthalmic
assessment.
Results-The results of the molecular
linkage study identifying a missense mu-
tation in the gene encoding the major
intrinsic protein of the lens (MIP) have
been published elsewhere. Affected indi-
viduals had bilateral discrete progressive
punctate lens opacities limited to mid and
peripheral lamellae with additional asym-
metric polar opacification. One young
female had predominantly cortical cata-
ract and another had serpiginous nuclear
opacities.
Conclusions-This phenotype has not
been recorded in human families before
and has been termed polymorphic. The
pattern of opacification appears to reflect
the distribution of MIP in the lens.

Abstract
Background-Congenital cataract, when
inherited as an isolated abnormality, is
phenotypically and genetically heteroge-
neous. Although there is no agreed no-
menclature for the patterns of cataract
observed, a recent study identified eight
readily identifiable phenotypes.
Methods-The Moorfields Eye Hospital
genetic eye clinic database was used to
identify a four generation family with iso-
lated autosomal dominant congenital
cataracts. All individuals (affected and
unaffected) underwent a full ophthalmic
assessment.
Results-The results of the molecular
linkage study identifying a missense mu-
tation in the gene encoding the major
intrinsic protein of the lens (MIP) have
been published elsewhere. Affected indi-
viduals had bilateral discrete progressive
punctate lens opacities limited to mid and
peripheral lamellae with additional asym-
metric polar opacification. One young
female had predominantly cortical cata-
ract and another had serpiginous nuclear
opacities.
Conclusions-This phenotype has not
been recorded in human families before
and has been termed polymorphic. The
pattern of opacification appears to reflect
the distribution of MIP in the lens.

Abstract
Background-Congenital cataract, when
inherited as an isolated abnormality, is
phenotypically and genetically heteroge-
neous. Although there is no agreed no-
menclature for the patterns of cataract
observed, a recent study identified eight
readily identifiable phenotypes.
Methods-The Moorfields Eye Hospital
genetic eye clinic database was used to
identify a four generation family with iso-
lated autosomal dominant congenital
cataracts. All individuals (affected and
unaffected) underwent a full ophthalmic
assessment.
Results-The results of the molecular
linkage study identifying a missense mu-
tation in the gene encoding the major
intrinsic protein of the lens (MIP) have
been published elsewhere. Affected indi-
viduals had bilateral discrete progressive
punctate lens opacities limited to mid and
peripheral lamellae with additional asym-
metric polar opacification. One young
female had predominantly cortical cata-
ract and another had serpiginous nuclear
opacities.
Conclusions-This phenotype has not
been recorded in human families before
and has been termed polymorphic. The
pattern of opacification appears to reflect
the distribution of MIP in the lens.

Abstract
Background-Congenital cataract, when
inherited as an isolated abnormality, is
phenotypically and genetically heteroge-
neous. Although there is no agreed no-
menclature for the patterns of cataract
observed, a recent study identified eight
readily identifiable phenotypes.
Methods-The Moorfields Eye Hospital
genetic eye clinic database was used to
identify a four generation family with iso-
lated autosomal dominant congenital
cataracts. All individuals (affected and
unaffected) underwent a full ophthalmic
assessment.
Results-The results of the molecular
linkage study identifying a missense mu-
tation in the gene encoding the major
intrinsic protein of the lens (MIP) have
been published elsewhere. Affected indi-
viduals had bilateral discrete progressive
punctate lens opacities limited to mid and
peripheral lamellae with additional asym-
metric polar opacification. One young
female had predominantly cortical cata-
ract and another had serpiginous nuclear
opacities.
Conclusions-This phenotype has not
been recorded in human families before
and has been termed polymorphic. The
pattern of opacification appears to reflect
the distribution of MIP in the lens.

Abstract
Background-Congenital cataract, when
inherited as an isolated abnormality, is
phenotypically and genetically heteroge-
neous. Although there is no agreed no-
menclature for the patterns of cataract
observed, a recent study identified eight
readily identifiable phenotypes.
Methods-The Moorfields Eye Hospital
genetic eye clinic database was used to
identify a four generation family with iso-
lated autosomal dominant congenital
cataracts. All individuals (affected and
unaffected) underwent a full ophthalmic
assessment.
Results-The results of the molecular
linkage study identifying a missense mu-
tation in the gene encoding the major
intrinsic protein of the lens (MIP) have
been published elsewhere. Affected indi-
viduals had bilateral discrete progressive
punctate lens opacities limited to mid and
peripheral lamellae with additional asym-
metric polar opacification. One young
female had predominantly cortical cata-
ract and another had serpiginous nuclear
opacities.
Conclusions-This phenotype has not
been recorded in human families before
and has been termed polymorphic. The
pattern of opacification appears to reflect
the distribution of MIP in the lens.

Abstract
Background-Congenital cataract, when
inherited as an isolated abnormality, is
phenotypically and genetically heteroge-
neous. Although there is no agreed no-
menclature for the patterns of cataract
observed, a recent study identified eight
readily identifiable phenotypes.
Methods-The Moorfields Eye Hospital
genetic eye clinic database was used to
identify a four generation family with iso-
lated autosomal dominant congenital
cataracts. All individuals (affected and
unaffected) underwent a full ophthalmic
assessment.
Results-The results of the molecular
linkage study identifying a missense mu-
tation in the gene encoding the major
intrinsic protein of the lens (MIP) have
been published elsewhere. Affected indi-
viduals had bilateral discrete progressive
punctate lens opacities limited to mid and
peripheral lamellae with additional asym-
metric polar opacification. One young
female had predominantly cortical cata-
ract and another had serpiginous nuclear
opacities.
Conclusions-This phenotype has not
been recorded in human families before
and has been termed polymorphic. The
pattern of opacification appears to reflect
the distribution of MIP in the lens.

Abstract
Background-Congenital cataract, when
inherited as an isolated abnormality, is
phenotypically and genetically heteroge-
neous. Although there is no agreed no-
menclature for the patterns of cataract
observed, a recent study identified eight
readily identifiable phenotypes.
Methods-The Moorfields Eye Hospital
genetic eye clinic database was used to
identify a four generation family with iso-
lated autosomal dominant congenital
cataracts. All individuals (affected and
unaffected) underwent a full ophthalmic
assessment.
Results-The results of the molecular
linkage study identifying a missense mu-
tation in the gene encoding the major
intrinsic protein of the lens (MIP) have
been published elsewhere. Affected indi-
viduals had bilateral discrete progressive
punctate lens opacities limited to mid and
peripheral lamellae with additional asym-
metric polar opacification. One young
female had predominantly cortical cata-
ract and another had serpiginous nuclear
opacities.
Conclusions-This phenotype has not
been recorded in human families before
and has been termed polymorphic. The
pattern of opacification appears to reflect
the distribution of MIP in the lens.

Abstract
Background-Congenital cataract, when
inherited as an isolated abnormality, is
phenotypically and genetically heteroge-
neous. Although there is no agreed no-
menclature for the patterns of cataract
observed, a recent study identified eight
readily identifiable phenotypes.
Methods-The Moorfields Eye Hospital
genetic eye clinic database was used to
identify a four generation family with iso-
lated autosomal dominant congenital
cataracts. All individuals (affected and
unaffected) underwent a full ophthalmic
assessment.
Results-The results of the molecular
linkage study identifying a missense mu-
tation in the gene encoding the major
intrinsic protein of the lens (MIP) have
been published elsewhere. Affected indi-
viduals had bilateral discrete progressive
punctate lens opacities limited to mid and
peripheral lamellae with additional asym-
metric polar opacification. One young
female had predominantly cortical cata-
ract and another had serpiginous nuclear
opacities.
Conclusions-This phenotype has not
been recorded in human families before
and has been termed polymorphic. The
pattern of opacification appears to reflect
the distribution of MIP in the lens.

Abstract
Background-Congenital cataract, when
inherited as an isolated abnormality, is
phenotypically and genetically heteroge-
neous. Although there is no agreed no-
menclature for the patterns of cataract
observed, a recent study identified eight
readily identifiable phenotypes.
Methods-The Moorfields Eye Hospital
genetic eye clinic database was used to
identify a four generation family with iso-
lated autosomal dominant congenital
cataracts. All individuals (affected and
unaffected) underwent a full ophthalmic
assessment.
Results-The results of the molecular
linkage study identifying a missense mu-
tation in the gene encoding the major
intrinsic protein of the lens (MIP) have
been published elsewhere. Affected indi-
viduals had bilateral discrete progressive
punctate lens opacities limited to mid and
peripheral lamellae with additional asym-
metric polar opacification. One young
female had predominantly cortical cata-
ract and another had serpiginous nuclear
opacities.
Conclusions-This phenotype has not
been recorded in human families before
and has been termed polymorphic. The
pattern of opacification appears to reflect
the distribution of MIP in the lens.

Abstract
Background-Congenital cataract, when
inherited as an isolated abnormality, is
phenotypically and genetically heteroge-
neous. Although there is no agreed no-
menclature for the patterns of cataract
observed, a recent study identified eight
readily identifiable phenotypes.
Methods-The Moorfields Eye Hospital
genetic eye clinic database was used to
identify a four generation family with iso-
lated autosomal dominant congenital
cataracts. All individuals (affected and
unaffected) underwent a full ophthalmic
assessment.
Results-The results of the molecular
linkage study identifying a missense mu-
tation in the gene encoding the major
intrinsic protein of the lens (MIP) have
been published elsewhere. Affected indi-
viduals had bilateral discrete progressive
punctate lens opacities limited to mid and
peripheral lamellae with additional asym-
metric polar opacification. One young
female had predominantly cortical cata-
ract and another had serpiginous nuclear
opacities.
Conclusions-This phenotype has not
been recorded in human families before
and has been termed polymorphic. The
pattern of opacification appears to reflect
the distribution of MIP in the lens.

Abstract
Background-Congenital cataract, when
inherited as an isolated abnormality, is
phenotypically and genetically heteroge-
neous. Although there is no agreed no-
menclature for the patterns of cataract
observed, a recent study identified eight
readily identifiable phenotypes.
Methods-The Moorfields Eye Hospital
genetic eye clinic database was used to
identify a four generation family with iso-
lated autosomal dominant congenital
cataracts. All individuals (affected and
unaffected) underwent a full ophthalmic
assessment.
Results-The results of the molecular
linkage study identifying a missense mu-
tation in the gene encoding the major
intrinsic protein of the lens (MIP) have
been published elsewhere. Affected indi-
viduals had bilateral discrete progressive
punctate lens opacities limited to mid and
peripheral lamellae with additional asym-
metric polar opacification. One young
female had predominantly cortical cata-
ract and another had serpiginous nuclear
opacities.
Conclusions-This phenotype has not
been recorded in human families before
and has been termed polymorphic. The
pattern of opacification appears to reflect
the distribution of MIP in the lens.

Abstract
Background-Congenital cataract, when
inherited as an isolated abnormality, is
phenotypically and genetically heteroge-
neous. Although there is no agreed no-
menclature for the patterns of cataract
observed, a recent study identified eight
readily identifiable phenotypes.
Methods-The Moorfields Eye Hospital
genetic eye clinic database was used to
identify a four generation family with iso-
lated autosomal dominant congenital
cataracts. All individuals (affected and
unaffected) underwent a full ophthalmic
assessment.
Results-The results of the molecular
linkage study identifying a missense mu-
tation in the gene encoding the major
intrinsic protein of the lens (MIP) have
been published elsewhere. Affected indi-
viduals had bilateral discrete progressive
punctate lens opacities limited to mid and
peripheral lamellae with additional asym-
metric polar opacification. One young
female had predominantly cortical cata-
ract and another had serpiginous nuclear
opacities.
Conclusions-This phenotype has not
been recorded in human families before
and has been termed polymorphic. The
pattern of opacification appears to reflect
the distribution of MIP in the lens.

Abstract
Background-Congenital cataract, when
inherited as an isolated abnormality, is
phenotypically and genetically heteroge-
neous. Although there is no agreed no-
menclature for the patterns of cataract
observed, a recent study identified eight
readily identifiable phenotypes.
Methods-The Moorfields Eye Hospital
genetic eye clinic database was used to
identify a four generation family with iso-
lated autosomal dominant congenital
cataracts. All individuals (affected and
unaffected) underwent a full ophthalmic
assessment.
Results-The results of the molecular
linkage study identifying a missense mu-
tation in the gene encoding the major
intrinsic protein of the lens (MIP) have
been published elsewhere. Affected indi-
viduals had bilateral discrete progressive
punctate lens opacities limited to mid and
peripheral lamellae with additional asym-
metric polar opacification. One young
female had predominantly cortical cata-
ract and another had serpiginous nuclear
opacities.
Conclusions-This phenotype has not
been recorded in human families before
and has been termed polymorphic. The
pattern of opacification appears to reflect
the distribution of MIP in the lens.

Abstract
Background-Congenital cataract, when
inherited as an isolated abnormality, is
phenotypically and genetically heteroge-
neous. Although there is no agreed no-
menclature for the patterns of cataract
observed, a recent study identified eight
readily identifiable phenotypes.
Methods-The Moorfields Eye Hospital
genetic eye clinic database was used to
identify a four generation family with iso-
lated autosomal dominant congenital
cataracts. All individuals (affected and
unaffected) underwent a full ophthalmic
assessment.
Results-The results of the molecular
linkage study identifying a missense mu-
tation in the gene encoding the major
intrinsic protein of the lens (MIP) have
been published elsewhere. Affected indi-
viduals had bilateral discrete progressive
punctate lens opacities limited to mid and
peripheral lamellae with additional asym-
metric polar opacification. One young
female had predominantly cortical cata-
ract and another had serpiginous nuclear
opacities.
Conclusions-This phenotype has not
been recorded in human families before
and has been termed polymorphic. The
pattern of opacification appears to reflect
the distribution of MIP in the lens.

Abstract
Background-Congenital cataract, when
inherited as an isolated abnormality, is
phenotypically and genetically heteroge-
neous. Although there is no agreed no-
menclature for the patterns of cataract
observed, a recent study identified eight
readily identifiable phenotypes.
Methods-The Moorfields Eye Hospital
genetic eye clinic database was used to
identify a four generation family with iso-
lated autosomal dominant congenital
cataracts. All individuals (affected and
unaffected) underwent a full ophthalmic
assessment.
Results-The results of the molecular
linkage study identifying a missense mu-
tation in the gene encoding the major
intrinsic protein of the lens (MIP) have
been published elsewhere. Affected indi-
viduals had bilateral discrete progressive
punctate lens opacities limited to mid and
peripheral lamellae with additional asym-
metric polar opacification. One young
female had predominantly cortical cata-
ract and another had serpiginous nuclear
opacities.
Conclusions-This phenotype has not
been recorded in human families before
and has been termed polymorphic. The
pattern of opacification appears to reflect
the distribution of MIP in the lens.

Abstract
Background-Congenital cataract, when
inherited as an isolated abnormality, is
phenotypically and genetically heteroge-
neous. Although there is no agreed no-
menclature for the patterns of cataract
observed, a recent study identified eight
readily identifiable phenotypes.
Methods-The Moorfields Eye Hospital
genetic eye clinic database was used to
identify a four generation family with iso-
lated autosomal dominant congenital
cataracts. All individuals (affected and
unaffected) underwent a full ophthalmic
assessment.
Results-The results of the molecular
linkage study identifying a missense mu-
tation in the gene encoding the major
intrinsic protein of the lens (MIP) have
been published elsewhere. Affected indi-
viduals had bilateral discrete progressive
punctate lens opacities limited to mid and
peripheral lamellae with additional asym-
metric polar opacification. One young
female had predominantly cortical cata-
ract and another had serpiginous nuclear
opacities.
Conclusions-This phenotype has not
been recorded in human families before
and has been termed polymorphic. The
pattern of opacification appears to reflect
the distribution of MIP in the lens.

Abstract
Background-Congenital cataract, when
inherited as an isolated abnormality, is
phenotypically and genetically heteroge-
neous. Although there is no agreed no-
menclature for the patterns of cataract
observed, a recent study identified eight
readily identifiable phenotypes.
Methods-The Moorfields Eye Hospital
genetic eye clinic database was used to
identify a four generation family with iso-
lated autosomal dominant congenital
cataracts. All individuals (affected and
unaffected) underwent a full ophthalmic
assessment.
Results-The results of the molecular
linkage study identifying a missense mu-
tation in the gene encoding the major
intrinsic protein of the lens (MIP) have
been published elsewhere. Affected indi-
viduals had bilateral discrete progressive
punctate lens opacities limited to mid and
peripheral lamellae with additional asym-
metric polar opacification. One young
female had predominantly cortical cata-
ract and another had serpiginous nuclear
opacities.
Conclusions-This phenotype has not
been recorded in human families before
and has been termed polymorphic. The
pattern of opacification appears to reflect
the distribution of MIP in the lens.

Abstract
Background-Congenital cataract, when
inherited as an isolated abnormality, is
phenotypically and genetically heteroge-
neous. Although there is no agreed no-
menclature for the patterns of cataract
observed, a recent study identified eight
readily identifiable phenotypes.
Methods-The Moorfields Eye Hospital
genetic eye clinic database was used to
identify a four generation family with iso-
lated autosomal dominant congenital
cataracts. All individuals (affected and
unaffected) underwent a full ophthalmic
assessment.
Results-The results of the molecular
linkage study identifying a missense mu-
tation in the gene encoding the major
intrinsic protein of the lens (MIP) have
been published elsewhere. Affected indi-
viduals had bilateral discrete progressive
punctate lens opacities limited to mid and
peripheral lamellae with additional asym-
metric polar opacification. One young
female had predominantly cortical cata-
ract and another had serpiginous nuclear
opacities.
Conclusions-This phenotype has not
been recorded in human families before
and has been termed polymorphic. The
pattern of opacification appears to reflect
the distribution of MIP in the lens.

Abstract
Background-Congenital cataract, when
inherited as an isolated abnormality, is
phenotypically and genetically heteroge-
neous. Although there is no agreed no-
menclature for the patterns of cataract
observed, a recent study identified eight
readily identifiable phenotypes.
Methods-The Moorfields Eye Hospital
genetic eye clinic database was used to
identify a four generation family with iso-
lated autosomal dominant congenital
cataracts. All individuals (affected and
unaffected) underwent a full ophthalmic
assessment.
Results-The results of the molecular
linkage study identifying a missense mu-
tation in the gene encoding the major
intrinsic protein of the lens (MIP) have
been published elsewhere. Affected indi-
viduals had bilateral discrete progressive
punctate lens opacities limited to mid and
peripheral lamellae with additional asym-
metric polar opacification. One young
female had predominantly cortical cata-
ract and another had serpiginous nuclear
opacities.
Conclusions-This phenotype has not
been recorded in human families before
and has been termed polymorphic. The
pattern of opacification appears to reflect
the distribution of MIP in the lens.

Abstract
Background-Congenital cataract, when
inherited as an isolated abnormality, is
phenotypically and genetically heteroge-
neous. Although there is no agreed no-
menclature for the patterns of cataract
observed, a recent study identified eight
readily identifiable phenotypes.
Methods-The Moorfields Eye Hospital
genetic eye clinic database was used to
identify a four generation family with iso-
lated autosomal dominant congenital
cataracts. All individuals (affected and
unaffected) underwent a full ophthalmic
assessment.
Results-The results of the molecular
linkage study identifying a missense mu-
tation in the gene encoding the major
intrinsic protein of the lens (MIP) have
been published elsewhere. Affected indi-
viduals had bilateral discrete progressive
punctate lens opacities limited to mid and
peripheral lamellae with additional asym-
metric polar opacification. One young
female had predominantly cortical cata-
ract and another had serpiginous nuclear
opacities.
Conclusions-This phenotype has not
been recorded in human families before
and has been termed polymorphic. The
pattern of opacification appears to reflect
the distribution of MIP in the lens.

Inherited cataract is the most common treatable cause of visual loss in children. ${ }^{1}$ When it occurs in isolation, it is most often transmitted as an autosomal dominant trait. ${ }^{2}$ Many different phenotypes are recognised but as yet no agreed classification exists. A recent study of families with isolated non-syndromic human inherited cataract ${ }^{3}$ recorded eight clearly distinguishable phenotypes - anterior polar, posterior polar, nuclear, lamellar, blue dot, coralliform, cortical, and pulverulent.

Inherited cataract is also genetically heterogeneous. Independent chromosomal loci have been mapped to $1 \mathrm{p} 36,1 \mathrm{q} 21-25,2 \mathrm{q} 33-35,13 \mathrm{q}$, 16q22.1, 17p, 17q11-12, 17q24, 21q, and 22q. A recessive form has been linked with the I-blood group locus and characterisation of balanced chromosomal translocations has identified further loci on $2 \mathrm{p} 22.3,14 \mathrm{q} 24$, and Xp (Table 1a). Underlying mutations are now known in the crystallin genes at the loci on $2 \mathrm{q}^{4}{ }^{4}$ $21 \mathrm{q}^{5}$ and $22 \mathrm{q},{ }^{6}$ the connexin genes on $1 \mathrm{q}^{78}$ and $13 \mathrm{q}^{9}$ and the developmental regulator gene PITX3 on $10 \mathrm{q}$ (Table 1b).

We have identified a family with autosomal dominant progressive lamellar opacification of the lens associated with anterior and posterior polar cataract. This "polymorphic" phenotype has not previously been recorded in human pedigrees. We have genetically linked this family to the long arm of chromosome 12 , to the locus 12q14, and have shown that in this and another genetically unrelated family, a mutation in the MIP gene underlies cataractogenesis. ${ }^{10}$ In this paper, we describe the clinical spectrum of the disease.

Spontaneously occurring dominant mutations in major intrinsic protein (MIP) have already been shown to produce heritable cataract traits in mice. The $C a t^{F_{r}}$ mouse has a splice site mutation that leads to cataract and wrinkling of the capsule. The lop mouse (lens opacity) has a missense mutation which leads to mistargeting of the protein and accumulation in the endoplasmic reticulum of the lens fibre cell. ${ }^{11}$ The underlying mechanisms by which each results in cataractogenesis remain to be elucidated. 


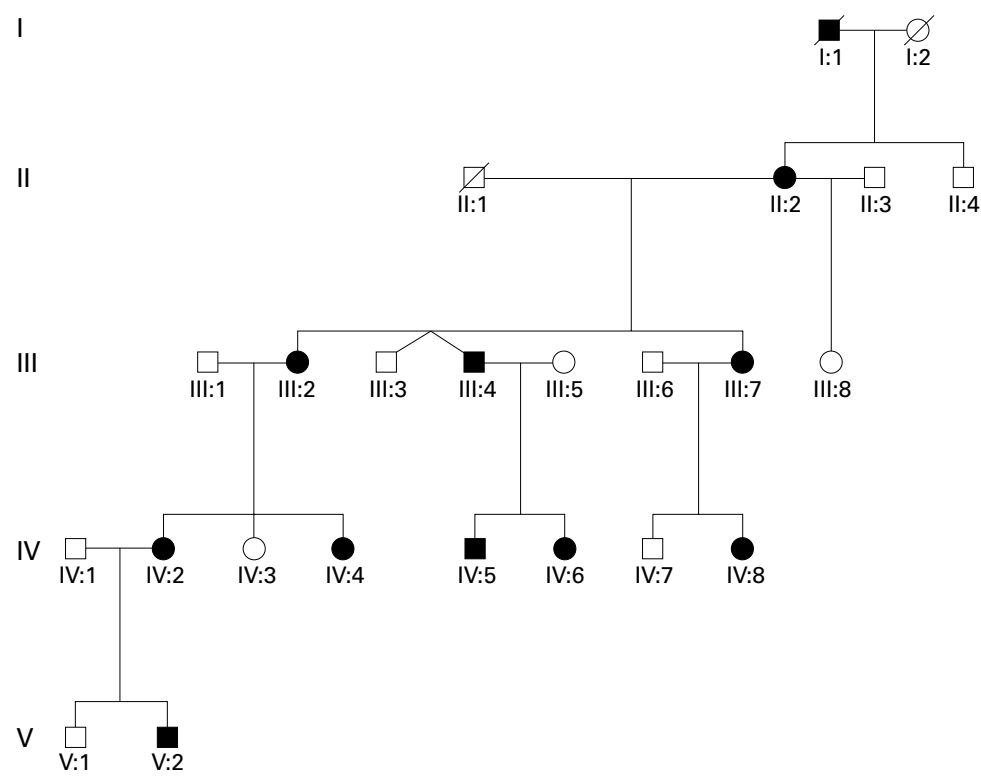

Figure 1 Abridged pedigree of family with polymorphic congenital cataract.

Patients and methods

The genetic database at Moorfields Eye Hospital provided details of the proband, from whom the pedigree was constructed and the family was invited to participate in the study. Ten affected and six unaffected members (Fig 1) underwent ophthalmological examination. Peripheral blood samples were taken for DNA extraction and the linkage study undertaken as previously described. ${ }^{12}$ Ethics committee approval was obtained for this study and patients gave their written consent to participate.

\section{Results}

The results of the molecular linkage study mapping the polymorphic cataract in this family to chromosome $12 \mathrm{q} 14$ and the subsequent detection of a mutation in the MIP gene have already been published. ${ }^{10}$

In the family, autosomal dominant inheritance was supported by the presence of affected male and female individuals in each generation and male to male transmission. The disease showed complete penetrance and highly variable expressivity.

Opacification of the lens was bilateral in all affected cases and consisted of discrete progressive punctate lens opacities limited to mid and peripheral lamellae. In addition, some of those affected had asymmetric anterior and posterior polar opacification. One young female had predominantly cortical cataract and another had serpiginous nuclear opacities. The clinical spectrum of the phenotype is shown in Figure 2. Hospital records indicated that the opacity was present at birth or developed within the first year of life. Visual acuity in the unoperated eyes of those affected ranged from $6 / 6$ to $6 / 24$ (Table 2). No affected individuals have developed strabismus or retinal detachment. There was no evidence of other ocular or systemic abnormalities.

Three individuals had undergone bilateral lensectomy in childhood, aged 1 month (left and right best corrected visual acuities 6/9,
$6 / 12$ respectively), 4 months $(6 / 6,6 / 9$; subsequently developed well controlled aphakic glaucoma), and 15 years (HM, 6/9). Right extracapsular cataract extraction had been performed on two patients in adulthood (aged 55 and 72 years, each achieving best corrected visual acuity of 6/6).

\section{Discussion}

In this paper, we document a new inherited cataract phenotype resulting from a mutation in the gene that encodes the major intrinsic protein of the lens (MIP), the most abundant membrane protein in the mature lens fibre cell.

A number of roles have been suggested for MIP but it is now known to be one of a number of sequence related proteins known as aquaporins, which form channels to selectively transport water molecules across cell membranes. ${ }^{13}$ The missense mutation we have described substitutes an amino acid thought critical for this specialised function.

Ten mammalian aquaporins are now known. ${ }^{14}$ Often more than one is expressed in a single tissue. The proteins are suggested to have important functions in the eye, being involved in aqueous humour dynamics and lacrimation. Aquaporins also have significant roles in renal water reabsorption, cerebrospinal fluid secretion, and the generation of pulmonary secretions have been suggested. ${ }^{15}$

To date, only aquaporin- 2 has been implicated in human disease. It is found abundantly in kidney collecting duct epithelium, where its function is regulated by $\mathrm{ADH}$ (antidiuretic hormone). Several missense mutations (recessive and dominant) have been identified that result in the development of nephrogenic diabetes insipidus. ${ }^{16}$ Interestingly, polymorphisms of aquaporin-1 (present on the red blood cell membrane, the Colton blood group antigens) have been documented that produce a non-functional protein (the null phenotype) but whose effects are subclinical. ${ }^{17}$ Such evidence has suggested that other mechanisms are able to compensate for aquaporin dysfunction. Our evidence that mutations in aquaporin-0 underlie cataract formation confirm the critical role of these proteins in certain tissues.

The phenotype of the cataract in this family has not previously been observed as an inherited trait $^{18}$ and to reflect the variable appearance between eyes and among individuals has been termed "polymorphic".

The discrete punctate opacities are similar to the pulverulent phenotype, which is characterised by fine dust-like opacification. However, the opacities in this pedigree are larger and, critically, their position within the lens is consistent contrasting markedly with the variable distribution of pulverulent opacities. Polar opacification is also not a feature of the pulverulent phenotype.

The phenotype observed in our family is also clearly distinct from the incompletely penetrant non-progressive polymorphic cataract mapped to chromosome 2q33-35 by Rogaev et $a l^{19}$ in the Turkmen populations of the former 
Soviet Union. In this cataract opacities resembled lumps of grapes or cotton anywhere from the fetal nucleus to the equator. Polar opacification was also not observed.

Although the mature lens fibre cell is metabolically inert, aquaporin water transport would be expected to continue as it is not energy dependent. Thus, the progressive na-
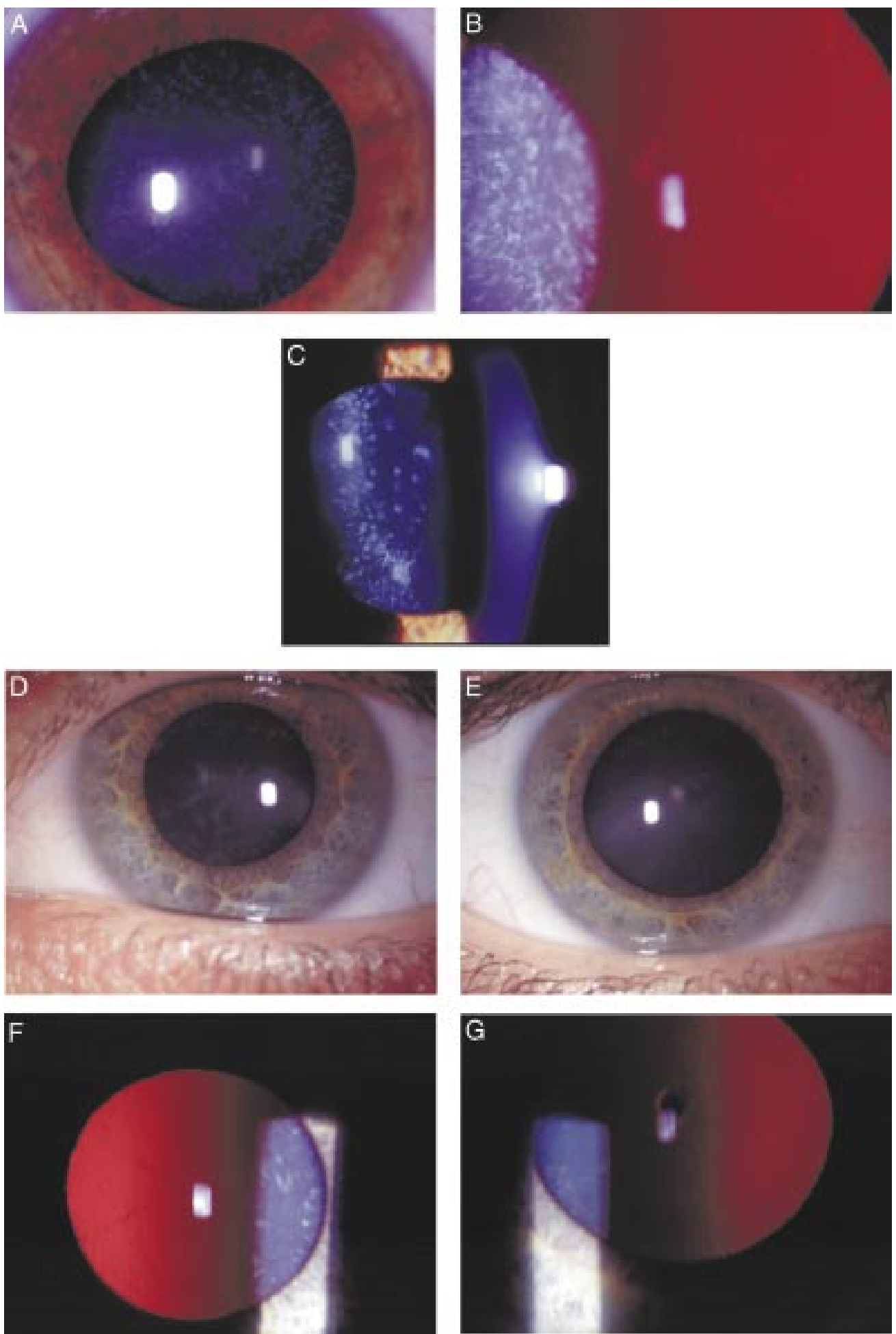

Figure 2 Punctate mid and peripheral opacities in: $(A)$ direct illumination (34 year old female); (B) retroillumination (same patient as $(A)) ;(C)$ slit beam illumination (17 year old female);(D) serpiginous nuclear opacities (24 year old female); (E) anterior polar cataract (same patient as in (D)); (F) retroillumination view of serpiginous nuclear opacities (same patient as in $(D)) ;(G)$ retroillumination view of anterior polar cataract in patient with predominantly cortical opacification. 
Table 2 Clinical data

\begin{tabular}{llll}
\hline & & \multicolumn{2}{l}{ Best corrected visual acuity } \\
\cline { 3 - 4 } $\begin{array}{l}\text { Affected } \\
\text { individual }\end{array}$ & $\begin{array}{c}\text { Age } \\
\text { (years) }\end{array}$ & Right & Left \\
\hline II:2 & 72 & $6 / 12$ & $6 / 6$ (pseudophakic) \\
III:2 & 55 & $6 / 24$ & $6 / 6$ (pseudophakic) \\
III:4 & 45 & $6 / 9$ (aphakic) & HM (aphakic) \\
III:7 & 45 & $6 / 9$ & $6 / 9$ \\
IV:2 & 35 & $6 / 6$ & $6 / 12$ \\
IV:4 & 27 & $6 / 6$ & $6 / 9-$ \\
IV:5 & 15 & $6 / 12$ (aphakic) & $6 / 9$ \\
IV:6 & 11 & $6 / 9$ (aphakic) & $6 / 6$ (aphakic) \\
IV:8 & 16 & $6 / 6$ & $6 / 6$ \\
V:2 & 1 & - & - \\
\hline
\end{tabular}

missense mutation in the MIP gene. ${ }^{10}$ Lens opacification in this family was nonprogressive and confirmed a perinuclear lamella. Such an observation is intriguing because it is not clear why two missense mutations in similar parts of the gene would produce such strikingly different phenotypes. Furthermore, this provides the first confirmation of allelic heterogeneity in this condition.

In conclusion, we have reported the identification of a new congenital cataract phenotype that results from a missense mutation in the major intrinsic protein of the lens. Studies of the implications of the mutation for the function of the protein will provide further understanding of how the underlying genotype relates to the phenotype observed.

The authors thank the family for their participation in this project and Mr Philip Ball (senior medical illustrator, Addenbrooke's Hospital, Cambridge) for his help in preparing the illustrations. This work was supported by a grant from the Wellcome Trust, 053416 to ATM and SSB.

1 Evans J, Rooney C, Ashwood F, et al. Blindness and partial sight in England and Wales: April 1990-March 1991. Health Trends 1996;28:5-12.

2 Francois J. Genetics of cataract. Ophthalmologica 1982;184: 61-71.

3 Ionides A, Francis P, Berry V, et al. Clinical and genetic heterogeneity in autosomal dominant congenital cataract. Br f Ophthalmol 1999;83:802-8.

4 Lubsen N, Renwick J, Tsui L-C, et al. A locus for human hereditary cataract is closely linked to the gamma-crystallin gene family. Proc Nat Acad Sci USA 1987;84:489-92.

5 Litt M, Kramer P, LaMorticella D, et al. Autosomal dominant congenital cataract associated with a missense mutation in the human alpha crystallin gene CRYAA. Hum Mol Genet 1998;7:471-4.

6 Litt M, Carrero-Valenzuela R, LaMorticella D, et al. AutoLitt M, Carrero-Valenzuela R, LaMorticella $\mathrm{D}$, et al. Auto-
somal dominant congenital cataract is associated with a somal dominant congenital cataract is associated with a chain termination mutation in the human be

7 Shiels A, Mackay D, Ionides A, et al. A missense mutation in the human connexin 50 gene (GJA8) underlies autosomal dominant "zonular pulverulent" cataract, on chromosome 1q. Am f Hum Genet 1998;62:526-32.

8 Berry V, Mackay D, Khaliq S, et al. Connexin 50 mutation in a family with congenital "zonular nuclear" pulverueln cataract (CZNP) of Pakistani origin. Hum Genet 1999;105 168-70.

9 Mackay D, Ionides A, Kibar Z, et al. Connexin46 mutations in autosomal dominant congenital cataract. Am 7 Hum Genet 1999;64:1357-64.
10 Berry V, Francis P, Kaushal S, et al. Missense mutations in the human $M I P$ gene, encoding the major intrinsic protein of the lens, underlie autosomal dominant "polymorphic" and lamellar cataracts on 12q. Nat Genet 2000;25:15-17.

11 Shiels A, Bassnett S. Mutations in the founder of the MIP gene family underlie cataract development in the mouse. Nat Genet 1996;12:212-15.

12 Ionides A, Berry V, Mackay D, et al. A locus for autosomal dominant posterior polar cataract on chromosome $1 \mathrm{p}$. Hum Mol Genet 1997;6:47-51.

13 Heymann J, Agre P, Engel A. Progress on the structure and function of aquaporin 1. F Struc Biol 1998;121:191-206.

14 Echevarria M, Ilundain A. Aquaporins. F Physiol Biochem 1998;54:107-18.

15 Lee M, King L, Agre P. The aquaporin family of water channel proteins in clinical medicine. Medicine (Baltimore) 1997;76:141-56.

16 Tamarappoo B, Yang B, Verkman A. Misfolding of mutant aquaporin-2 water channels in nephrogenic diabetes insipidus. F Biol Chem 1999;274:34825-31.

17 Daniels G. Functional aspects of red cell antigens. Blood Rev 1999;13:14-35.

18 Yanoff M, Duker J. Ophthalmology. St Louis: Mosby International, 1999

19 Rogaev E, Rogaeva E, Korovaitseva G, et al. Linkage of polymorphic congenital cataract to the gamma-crystallin ocus on human chromosome 2q33-35. Hum Mol Genet 1996;5:699-703.

20 Eiberg H, Lund A, Warburg $M$, et al. Assignment of congenital cataract Volkmann-type (CCV) to chromosome 1p36. Hum Genet 1995;96:33-8.

21 American Society of Genetics. Identification of an autosomal dominant cataract locus on chromosome $12 \mathrm{q} 12$ 12q14.1. 1998.

22 Moross T, Vaithilingam S, Styles S, et al. Autosomal dominant anterior polar cataracts associated with a familial $2 ; 14$ translocation. F Med Genet 1984;21:52-3.

23 Yokoyama Y, Narahara K, Tsuji K, et al. Autosomal dominant congenital cataract and microphthalmia associated with a familial t $(2 ; 16)$ translocation. Hum Genet 1992; 90:177-8.

24 Eiberg E, Marner E, Rosenberg T, et al. Marner's cataract (CAM) assigned to chromosome 16: linkage to haptoglobin. Clin Genet 1988;34:272-5.

25 Berry V, Ionides A, Moore A, et al. A locus for autosomal dominant anterior polar cataract on chromosome $17 \mathrm{p} . \mathrm{Mol}$ Genet 1996;5:415-19.

26 Padma T, Ayyagari R, Murty J, et al. Autosomal dominant zonular cataract with sutural opacities localised to chromosome 17q11-12. Am 7 Hum Genet 1995;57:850-5.

27 Armitage M, Kivlin J, Ferrel R. A progressive early onset cataract gene maps to human chromosome 17q24. Nat Genet 1995;9:37-40.

28 Ogata H, Okubo Y, Akabara T. Phenotype i associated with congenital cataract in Japanese. Transfusion 1979;19:166-8.

29 Krill A, Woodbury G, Bowman J. X-chromosomal-linked sutural cataracts. Am f Ophthalmol 1969;68:867-72.

30 Shambolian D, Lewis R, Buctow K, et al. Nance-Horan syndrome: localisation within the region Xp22.1-22.3 by linkage analysis. Am f Hum Genet 1990;47:13-19.

31 Warburg $M$. X-linked cataract and X-linked microphthalmos: how many deletion families? Am 7 Med Genet 1989;34:451-3.

32 Stephan D, Gillanders E, Vanderveen D, et al. Progressive juvenile-onset punctate cataract characterised by mutation of the gamma-D-crystallin gene. Proc Natl Acad Sci USA 1999;96:1008-12.

33 Heon E, Priston M, Schorederet D, et al. The gammacrystallins and human cataracts: a puzzle made clearer. $\mathrm{Am}$ 7 Hum Genet 1999;65:1261-7.

34 Semina E, Ferrell R, Mintz-Hittner H, et al. A novel homeobox gene PITX3 is mutated in families with autosomal dominant cataracts and ASMD. Nat Genet 1998;19:16770 .

35 Kramer P, Yount J, Michell T, et al. A second gene for cerulean cataract maps to the beta-crystallin region on chromosome 22. Genomics 1996;35:539-42.

36 Gill D, Klose R, Munier F, et al. Genetic heterogeneity of the Coppock-like cataract: a mutation in CRYBB2 on chromosome 22q11.2. Invest Ophthalmol Vis Sci 2000;41: $159-65$. 